in an average expert system makes the system untestable; at least if testability means that every potential pathway be tested. The expert system that flies your 'plane home from holiday is 'backed-up' by having a pilot on board. Hopefully, if the expert system decides to nosedive your 'plane into the ground, the pilot will take over the controls!

So what is the potential consequence for psychiatric research? Image analysis, done by computers, relies on expert systems. These are untestable. The very least we need to do is analyse our brain scans using two completely independent software programs. If, and only If, the two software systems produce the same results then we may be able to draw significant conclusions.

The problem, however, does not stop there. The scans themselves are produced by computer guided equipment. Serial scans by different machines have already been criticised in the literature. If results between research centres are to be comparable, then at least two scans per patient, done on different machines, and analysed by two different software packages may be needed. That makes four analyses per patient mandatory, whether or not the experimental design uses a 'case' v. 'control' methodology or not.

BILDER R.M. et al, (1994) Schizophrenia Research, 11, 131.

STUART COX UCL/Middlesex Hospital; ANN MORTMER Charing Cross Hospital, London W6 8RF and PAUL JACKSON Bioinformatics Department, UCL/Middlesex Hospital, London WIN 8AA

\section{Overuse of hypnotics for psychiatric in-patients}

Sir: There still appears to be a widespread problem with hypnotic prescription for psychiatric in-patients despite advice from the British National Formulary (1994) and the Royal College of Psychiatrists (1988) which both state that hypnotics should not be routinely prescribed for in-patients unless there are specific indications.

Insomnia causes patients subjective distress but is not dangerous. Many psychiatric illnesses present with insomnia which does not usually need either immediate treatment or treatment in isolation from other symptoms. Treating insomnia too early can mask illness during assessment causing confusion in diagnosis. Hypnotics are addictive and expensive, and form a large proportion of the NHS drug budget on volume of prescription alone. Hypnotics should therefore not be indiscriminately prescribed.

Hypnotics are often prescribed by an on call doctor in the middle of the night or late evening for whom a significant motivation in prescribing was in not being disturbed by nursing staff further (Fry, 1985). Night sedation was also frequently started on the night of admission. However, as the duty doctor is not the key medical contact and often has inadequate knowledge of the patient's background and treatment plan, prescription of hypnotics by him or her is frequently inappropriate.

The solutions to this problem lie in proper education of staff and patients, in good communication and in consistency of treatment. Key points are:

(a) The patient needs to be educated that it is reasonable to expect initial insomnia in the early days of admission

(b) nurses may need reminding of simple management of mild insomnia with light physical exercise during the day, reducing caffeine intake in the evenings and reassuring some patients once their anxieties are understood

(c) duty medical staff should observe consistency of treatment and not feel pressurised by staff or patients to prescribe hypnotics inappropriately

(d) medical staff directly responsible for the patient's care must be explicit to other staff and patients about their policy on prescription of hypnotics for their in-patients.

Prescribing costs can be reduced and quality of care improved if hypnotics are prescribed appropriately. They should be prescribed mainly by the patient's own doctor, unless insomnia is "severe, disabling or subjecting the individual to extreme distress" (BNF, 1994). Nursing staff have an important role in containing patients anxieties and in informing and educating them about appropriate management of their insomnia.

Britsh Medical association and the Pharmaceutical SOCIETY (1994) British National Formulary, 27, 138.

FRY R.P.W. (1985) Night sedation in the admission wards of a psychiatric hospital. Psychiatric Bulletin. 13, $184-185$.

Royal College of Psychiatrists (1988) Benzodiazepines and dependence: a College statement. Bulletin of the Royal College of Psychiatrists, 12, 107-108.

ALBERTO ALBENIZ and NICK STUART-SMITH, St Crispin Hospital, Duston, Northampton NN5 4UN

\section{Educating non-medical staff about the use of psychiatric drugs}

Sir: The role of the psychiatric registrar in learning disability is constantly evolving, as patients are relocated from hospital sites into small group homes within the community. Many 